\title{
Uma Abordagem Ontológica Aplicada ao Gerenciamento de Informação de Redes de Transporte Coletivo
}

\author{
Alternative title: An Ontological Approach for Information Management of \\ Public Transport Networks
}

\author{
Ernesto Fonseca Veiga \\ Instituto de Informática \\ Universidade Federal de Goiás \\ Goiânia, Brasil \\ ernestofonseca@inf.ufg.br
}

\author{
Renato de Freitas Bulcão-Neto \\ Instituto de Informática \\ Universidade Federal de Goiás \\ Goiânia, Brasil \\ renato@inf.ufg.br
}

\begin{abstract}
RESUMO
Este artigo contribui com pesquisas em desenvolvimento de sistemas de transporte inteligentes ao propor uma abordagem baseada em ontologias e especificações padrão da Web Semântica. A metodologia utilizada inclui a criação de um modelo ontológico com representação de informações espaçotemporais e a validação desse modelo por meio do desenvolvimento de um sistema de gerenciamento de transporte coletivo urbano de uma metrópole brasileira. Os autores defendem que a utilização de ontologias e especificações padrão de intercâmbio e consulta da Web Semântica facilita o desenvolvimento de sistemas de transporte inteligente quanto à representação semântica homogênea e compartilhada entre aplicações.
\end{abstract}

\section{Palavras-Chave}

Sistema de transporte inteligente, ontologia, Web Semântica.

\begin{abstract}
This paper presents an approach based on ontologies and Semantic Web specifications for information management of intelligent transport systems. The methodology includes the creation of an ontology-based model for representing spatiotemporal information as well as the model validation by means of the development of an urban transportation management system for a Brazilian metropolis. The authors argue that the combined use of ontologies and Semantic Web standard specifications for interchange and query makes it easier the development of intelligent transport systems concerning homogeneous and shared semantic representation among applications.
\end{abstract}

Permission to make digital or hard copies of all or part of this work for personal or classroom use is granted without fee provided that copies are not made or distributed for profit or commercial advantage and that copies bear this notice and the full citation on the first page. To copy otherwise, to republish, to post on servers or to redistribute to lists, requires prior specific permission and/or a fee.

SBSI 2015, May 26-29, 2015, Goiânia, Goiás, Brazil

Copyright SBC 2015.

\section{Categories and Subject Descriptors}

World Wide Web [Web data description languages]: Web Ontology Language (OWL); Information systems applications [Spatial-temporal systems]: [Location based services]

\section{General Terms}

Management, Design, Languages, Standardization.

\section{Keywords}

Intelligent transport system, ontology, Semantic Web.

\section{INTRODUÇÃO}

De acordo com os resultados do censo demográfico de 2010 , divulgado pelo IBGE ${ }^{1}$, e com o comparativo dos censos anteriores, o número de pessoas que residem em áreas urbanas está em constante crescimento, representando $84 \%$ da população brasileira. Apesar disso, a infraestrutura urbana não tem acompanhado esse ritmo de crescimento populacional, refletindo em prejuízo à qualidade de vida dos cidadãos. Dentre os principais problemas advindos deste crescimento está a mobilidade urbana, devido ao aumento da quantidade de veículos em circulação nas médias e grandes cidades, causando sobrecarga das vias e engarrafamentos [9].

Uma parte da solução para este problema é o investimento no transporte em massa, o qual em grande parte é representado pelo transporte coletivo urbano. Esta modalidade de transporte, na maioria das cidades que o possuem, é realizado através de ônibus, e devido à baixa qualidade e ineficiência do serviço prestado, que possui problemas como superlotações e atrasos, não atrai os usuários e principalmente os que possuem veículos de transporte particular.

Além da infraestrutura física para este tipo de serviço, a infraestrutura computacional tem se mostrado cada vez mais necessária e indispensável, tendo como exemplo principal a implantação dos Serviços de Transporte Inteligentes, ou Intelligent Transport Systems (ITS) [12]. Estes sistemas visam a eficiência dos serviços prestados, com o objetivo de

\footnotetext{
${ }^{1}$ Instituto Brasileiro de Geografia e Estatística: http://www.ibge.gov.br/home/mapa_site/mapa_site. php\#populacao
} 
melhorar a interação com os usuários, fornecendo-lhes informações de relevância a respeito do serviço, tais como horários, tempo de espera, duração da viagem, locais atendidos, entre outras.

Portanto, as informações relativas ao tráfego urbano estão intrinsecamente ligadas às noções de espaço e de tempo. Uma informação espaço-temporal se aplica a todo evento que acontece no mundo real, uma vez que estes eventos ocorrem em algum lugar do espaço e em um determinado momento no tempo [6]. Por esta razão, todo sistema de software desenvolvido para resolver um problema do mundo real, independente de seu propósito, trata um ou mesmo estes dois aspectos (espaço e tempo) em algum grau.

A representação e utilização de informação espaço-temporal é atualmente uma linha de pesquisa ativa, que tem ganhado importância em áreas, como a Web Semântica [1]. Especificações padrão da Web Semântica (OWL, RDF e SPQRQL) mostram-se como fortes candidatos para tratarem o intercâmbio, a modelagem e a consulta de informações espaçotemporais. Além disso, ferramentas baseadas nesses padrões oferecem um suporte poderoso à representação e ao gerenciamento de informações espaço-temporais.

Este artigo propõe uma abordagem que utiliza ontologias e padrões da Web Semântica para apoio à modelagem, representação e consulta de dados espaço-temporais. São apresentados o modelo OntoBus, construído com base em ontologias de referência, cada qual para representar informações de espaço e tempo, bem como o desenvolvimento de um sistema de informação de redes de transporte coletivo como prova de conceito, os quais representam as principais contribuições deste trabalho.

Os autores defendem que uma solução ontológica como a apresentada serve de base para o desenvolvimento de sistemas de transporte inteligentes, com ganhos quanto à expressividade e capacidade de inferência a partir de informação espaço-temporal, além da interoperabilidade semântica entre diferentes aplicações que fazem uso de informações de sistemas dessa natureza.

O artigo está assim organizado: a Seção 2 discorre sobre a fundamentação teórica e a metodologia utilizadas nesta pesquisa; a Seção 3 descreve a abordagem ontológica proposta aplicada ao domínio de transporte coletivo, bem como uma avaliação dos resultados; a Seção 4 apresenta trabalhos relacionados e a Seção 5, as conclusões e trabalhos futuros.

\section{MATERIAIS E MÉTODOS}

Esta seção apresenta os principais padrões, tecnologias e ontologias da Web Semântica utilizados neste trabalho. Em seguida, é apresentada também a metodologia adotada na realização desta pesquisa.

\subsection{Tecnologias e Padrões da Web Semântica}

Na Web Semântica, a representação de informação ganha ainda mais importância, uma vez que a modelagem de conceitos e objetos deve ser realizada com o devido rigor, para que seja possível reutilizar estes dados em diferentes aplicações [6]. Um modelo semântico de informações contém, além destas próprias, as regras de negócio para um domínio específico, tornando este tipo de modelo uma fonte de conhecimento compartilhado, que necessita de formalidade e consenso especializado para ser estruturado.

Informações semânticas são representadas como um conjunto de assertivas, denominadas declarações (ou statements) descritas como triplas, por serem estruturas compostas de três elementos: sujeito, predicado e objeto. Uma tripla pode ser representada como um grafo direcionado, onde o sujeito e o objeto são representados por nós que são ligados por uma aresta, representando o predicado. Este modelo de dados adotado na Web Semântica é parte integrante do padrão de intercâmbio de informação na Web Semântica, denominado Resource Description Framework ${ }^{2}$ (RDF) [6].

Os nós que compõem um grafo RDF (sujeitos e objetos) podem ser de dois tipos distintos: recursos e literais. Literais representam valores concretos de dados, como números e strings, e são exclusivamente objetos de triplas, e nunca sujeito destas. Os recursos, no entanto, representam qualquer tipo de informação, e podem ser tanto sujeitos quanto objetos das triplas. Já os predicados podem ser uma característica do sujeito, ou um relacionamento com um objeto.

No modelo de triplas do padrão RDF, um recurso pode representar qualquer coisa que possa ser nomeada. Dessa forma, um recurso é um nome que representa um objeto, uma ação ou um conceito. Para nomear um recurso são utilizadas Internationalized Resource Identifiers (IRI's), uma extensão do padrão Uniform Resource Identifier (URI), uma sequência de caracteres que permite nomear recursos de maneira única e não ambígua, ou seja, não existem recursos diferentes com uma mesma IRI [6].

A Figura 1 apresenta um grafo desconexo, gerado a partir de um arquivo RDF, que contém 4 (quatro) triplas. Este grafo descreve um recurso Bus1, do tipo Bus (ônibus), e um recurso BusStop1, do tipo BusStop (ponto de ônibus). Cada um desses recursos é sujeito de duas triplas, que têm como objetos um recurso (que representa o tipo do indivíduo por meio da propriedade type) e um valor literal (o identificador do ônibus e do ponto de ônibus, de acordo com o tipo do indivíduo). Esta figura foi gerada utilizando a ferramenta $R D F$ Validation Service do W3C ${ }^{3}$

Um modelo de triplas RDF pode ser serializado em diferentes formatos, que incluem XML, Turtle, N3 e JSON [6]. Por critérios de legibilidade e concisão, adotou-se neste trabalho o formato Turtle para demostrar os exemplos e trechos de modelos desenvolvidos. O grafo apresentado na Figura 1, por exemplo, foi gerado a partir de um modelo RDF que contém o seguinte conteúdo na forma de triplas:

$\begin{array}{llll}1 & \text { Oprefix bus: } & <\text { http://bus\#> } \\ 2 & \text { bus:Bus1 } & \text { a } & \text { bus:Bus } \\ 3 & & \text { bus:id } & \text { "263" } \\ 4 & \text { bus:BusStop1 } & \text { a } & \text { bus:BusStop ; } \\ 5 & & \text { bus:name } & \text { "31502" }\end{array}$

Na linha 1, tem-se a diretiva @prefix, que declara uma abreviação para um espaço de nomes que será utilizado no modelo. Na linha 2 é descrita a primeira tripla do modelo, que tem como sujeito o recurso Bus1. Esta tripla descreve a propriedade tipo, ou seja, a que classe pertence o recurso, representada pelo predicado a (é um), que liga o sujeito Bus1 ao objeto da tripla, no caso, Bus.

Quando uma linha se encerra com um ponto e vírgula (;) a linha seguinte descreve uma tripla que tem como sujeito o mesmo da linha anterior, neste caso o recurso Bus1. A propriedade descrita nesta tripla é a id, cujo valor é o objeto literal 263, como mostra a linha 3. As linhas 4 e 5, a exemplo

${ }^{2}$ RDF: http://www.w3.org/RDF/

${ }^{3}$ Validation Service: http://www .w3.org/RDF/Validator/ 


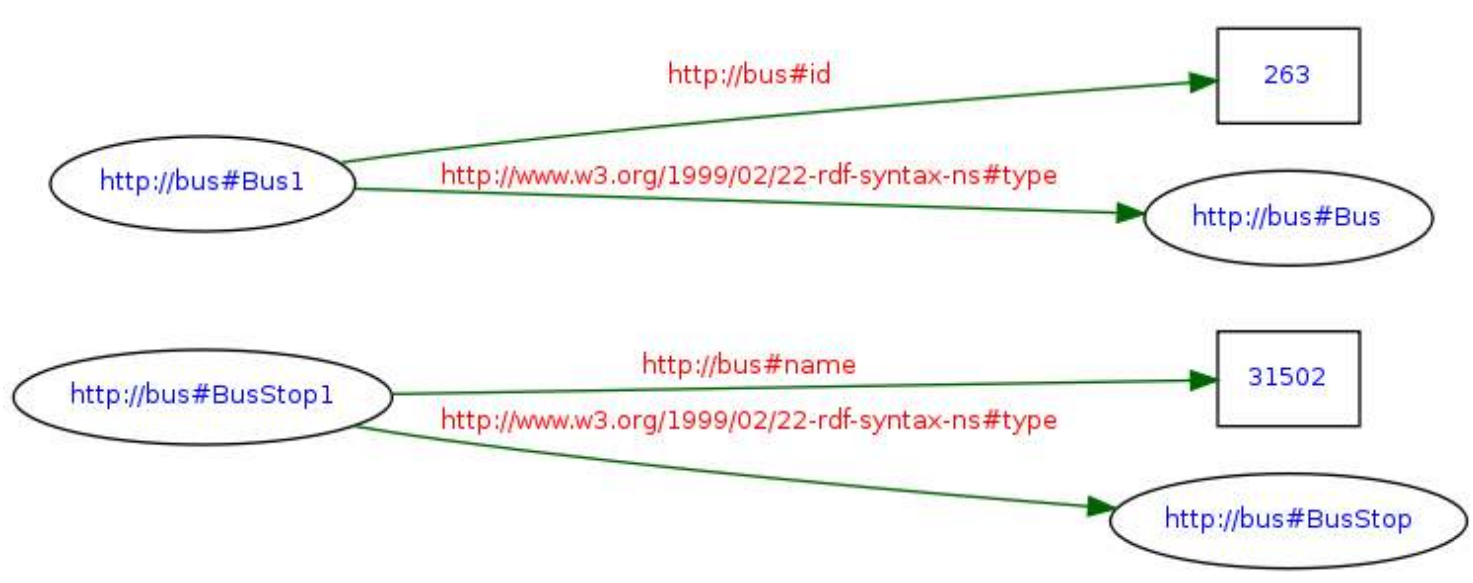

Figura 1: Grafo com 4 triplas, descrevendo um ônibus e sua linha.

do que foi descrito, descrevem duas triplas que têm como sujeito o recurso BusStop1, do tipo BusStop.

Discutida a maneira como se representa informações utilizando o modelo de triplas RDF e a serialização Turtle, o próximo passo é entender como consultar informações na Web Semântica. Para consultar um modelo de triplas RDF, adota-se a recomendação padrão W3C, chamada linguagem SPARQL (SPARQL Protocol and RDF Query Language ${ }^{4}$ [2].

O exemplo de consulta SPARQL apresentado a seguir é aplicado ao modelo de triplas supracitado. Essa consulta visa buscar o identificador de qualquer recurso que possua a propriedade bus:id, que são todos os recursos que pertencem à classe Bus.

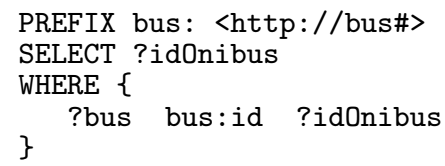

De acordo com o modelo de triplas RDF, esta consulta retorna apenas um resultado, pois há somente uma tripla que satisfaz as condições buscadas, como ilustrado a seguir.

$$
\begin{aligned}
& \text { | idOnibus | } \\
& \begin{array}{l}
=========== \\
\text { | "263" | }
\end{array}
\end{aligned}
$$

Os padrões RDF, para representação e intercâmbio de informação, e SPARQL, para consulta de informação em triplas RDF, foram abordados de maneira sucinta neste trabalho. Mais detalhes são encontrados no sítio do $\mathrm{W}^{3} \mathrm{C}^{5}$.

Embora a linguagem RDF forneça meios para representação de informação na Web Semântica, ela não é capaz de declarar o significado dessa informação. Para expressar semântica devem ser utilizadas ontologias, que incluem estruturas de representação capazes de expressar classes de entidades de um dado domínio, seus relacionamentos e axiomas, permitindo o processamento semântico das informações e uma interpretação formal dos dados [11].

Segundo [10], uma ontologia deve permitir o compartilhamento do entendimento comum das estruturas de informação, seja entre pessoas ou agentes de software; o reúso de

\footnotetext{
${ }^{4}$ SPARQL: http://www .w3.org/TR/rdf-sparql-query/ ${ }^{5}$ W3C: http: //www.w3.org/
}

informações de um domínio de conhecimento; a definição de premissas explícitas sobre um domínio; e a separação entre conhecimento de domínio e conhecimentos operacionais.

Para modelar domínios de conhecimento, desenvolvendo ontologias, a Web Semântica utiliza a linguagem de ontologia Web Ontology Language 6 (OWL), recomendada como padrão pelo W3C. Esta linguagem adiciona restrições a respeito da estrutura e do conteúdo dos documentos RDF, no intuito de permitir que o processamento e o raciocínio sobre esse conteúdo possam ser realizados por computadores.

A subseção seguinte apresenta dois exemplos de ontologias existentes para modelagem de informação espaço-temporal, necessárias para o desenvolvimento deste trabalho.

\subsection{Ontologias de Espaço e Tempo}

Para sistemas de transporte inteligentes, a principal informação espacial a ser considerada é a localização, que pode ser fixa, como no caso dos locais de parada, estações e terminais de embarque e desembarque, ou dinâmica, no caso da localização dos veículos de transporte, como ônibus, trens e metrô, em movimento na maior parte do tempo.

O Basic Geo Vocabulary, também conhecido como WGS84, é um vocabulário RDF desenvolvido pelo W3C para fornecer representação de informações espaciais tais como latitude, longitude e outras informações relacionadas. Este vocabulário inicia a exploração das possibilidades de representação de mapeamento e localização em RDF. Mantendo uma estrutura simples, o vocabulário WGS84 possibilita não somente descrever mapas, mas também as entidades que estão posicionadas em determinadas localizações nestes mapas [13].

A Figura 2 apresenta as principais classes do vocabulário WGS84: SpacialThing e Point. A classe Point (ponto), subclasse de SpacialThing, possui propriedades para representar latitude (geo:lat), longitude (geo:long) e altitude (geo:alt). Essas propriedades podem ser utilizadas de maneira independente e também associadas a propriedades definidas externamente, por outro vocabulário ou ontologia, para descrever uma entidade.

A partir dessas classes e de suas propriedades é possível relacionar uma entidade (e.g. um ônibus) a um ponto específico, e inclusive manter um registro da movimentação ou evolução de um objeto dinâmico no espaço. Essa caracte-

${ }^{6}$ OWL: http://www.w3.org/TR/owl-features/ 


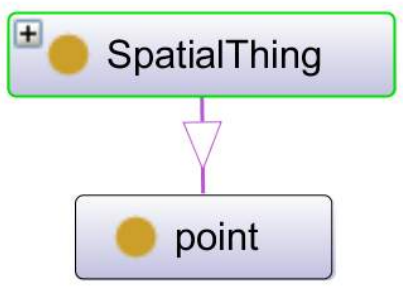

Figura 2: Principais classes do vocabulário WGS84.

rística é importante e pode ser utilizada em aplicações para o reconhecimento de padrões ou previsões de movimentação ou deslocamento.

A escolha do vocabulário WGS84 deu-se em função de sua simplicidade e adequação na representação de informações espaciais, como a localização de um ponto no espaço, para sistemas de transporte inteligentes.

Também relevante para sistemas de transporte inteligentes, a informação temporal possui facetas distintas, podendo ser pré-definida, como previsões de horários em que veículos estarão em determinado ponto ou estação, ou informações em tempo real da localização desses veículos.

Para a representação de informação temporal foi escolhida a ontologia $O W L$-Time, que descreve os aspectos temporais de uma entidade, apoiando a expressividade de relações topológicas entre momentos no tempo e intervalos de tempo, bem como horários e datas [8]. A Figura 3 apresenta as principais classes da ontologia OWL-Time: a super-classe TemporalEntity e suas subclasses Instant (instante de tempo) e Interval (intervalo de tempo).

Além da relação hierárquica entre as classes (setas contínuas), a figura ainda mostra relações entre classes (setas tracejadas). A relação entre as classes TemporalEntity e subclasse Instant expressa que toda entidade temporal possui instantes de início e de término, determinados pelas propriedades hasBeginning e hasEnd, respectivamente. Da mesma forma, a relação inside expressa que um dado instante Instant pertence a um intervalo de tempo Interval.

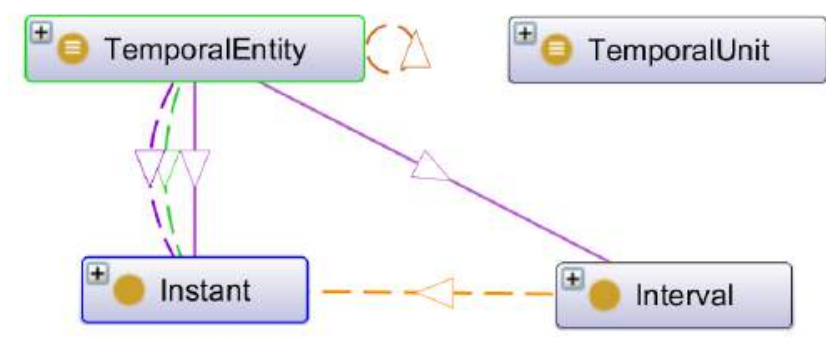

Figura 3: Principais classes da ontologia $O W L$-Time.

A ontologia $O W L$-Time também permite a utilização de unidades de tempo como segundos, minutos, horas, dias, dentre outras, relacionadas à classe TemporalUnit. Outras propriedades importantes neste contexto são before e after, que expressam se um evento ocorreu antes ou depois de outro, contribuindo para a realização de inferências neste domínio. Múltiplos tipos de relacionamentos podem também ser expressos entre eventos intervalares, como antecedência e sucessão imediatas, pertinência temporal, entre outros.
A $O W L$-Time foi adotada neste trabalho por oferecer uma representação completa e, ao mesmo tempo, concisa dos conceitos relacionados à semântica temporal. As informações aqui apresentadas são apenas o essencial para a compreensão da $O W L$-Time, sendo as definições completas desta ontologia encontradas em [7]. As figuras apresentadas nesta subseção foram geradas no editor de ontologias Protégé 7 .

\subsection{Metodologia adotada}

A metodologia seguida pelos autores envolve, de forma resumida, os seguintes passos:

1. Definição de requisitos para a construção de um modelo mínimo de informação para sistemas de transporte inteligente (ITS), dentre os quais, destacam-se:

- associação de informação espaço-temporal a entidades de um ITS;

- alta expressividade na descrição de informação espaço-temporal de entidades de um ITS;

- descrição de conhecimento consensual sobre informação espaço-temporal;

- compartilhamento de informações entre aplicações, que não sejam apenas ITS;

- validação formal para garantir a consistência do modelo e as instâncias que representa.

Segundo a visão dos autores, reforçada pela literatura [4], ontologias e especificações padrão da Web Semântica, como RDF, SPARQL e OWL, atendem aos requisitos de expressividade, formalidade, interoperabilidade e conhecimento consensual supracitados para um modelo de informação de um ITS.

2. Estudo de especificações padrão da Web Semântica:

- modelo de triplas RDF, formato padrão de intercâmbio de dados na Web Semântica;

- sintaxe Turtle de serialização de triplas RDF;

- linguagem SPARQL para consulta de dados expressos em RDF;

- linguagem OWL para construção de ontologias.

3. Estudo de ontologias que descrevem informação espaçotemporal:

- Seleção das ontologias WGS84 e OWL-Time;

- Reúso das ontologias WGS84 e OWL-Time, atividade sugerida pela Methodology 101 descrita em [10], principalmente quando já existem modelos ontológicos que tratam o contexto desejado.

4. Desenvolvimento do modelo OntoBus ${ }^{8}$ :

- Definição de conceitos específicos do modelo, como as classes Bus e BusStop;

- Associação de informação espaço-temporal das ontologias reusadas a conceitos específicos do modelo OntoBus;

- Validação do modelo OntoBus por meio de uma aplicação que descreve a evolução espaço-temporal de linhas de transporte coletivo.

${ }^{7}$ Protégé: http://protege.stanford.edu/

${ }^{8}$ Conjunto de atividades descritas na próxima seção. 


\section{ESTUDO DE CASO}

Neste estudo de caso foi realizada a representação da evolução espacial e temporal de ônibus que cumprem o trajeto da linha 263 da rede metropolitana de transporte coletivo de uma metrópole brasileira. Esta linha foi escolhida por ser uma das principais linhas de acesso ao campus universitário em que trabalham os autores desta pesquisa.

Para essa finalidade, coletamos dados sobre a linha 263 e criamos sua representação semântica. A Figura 4(a) apresenta os horários dos ônibus para dias úteis, sábados, domingos e feriados; e a Figura 4(b) apresenta o mapa do trajeto, com destaque para todos os pontos de parada para embarque e desembarque de usuários dos ônibus da linha 263.

\subsection{Modelo Ontológico OntoBus}

Através da utilização de ontologias de espaço e tempo, apresentamos neste estudo de caso o modelo semântico OntoBus, criado para o gerenciamento da informação de redes de transporte coletivo, instanciando como exemplo, a linha 263. Esta linha específica foi escolhida para demonstrar a utilização do OntoBus, mas a mesma representação pode ser estendida e/ou replicada para as demais linhas desta e de outras redes, sendo um modelo genérico.

As principais ferramentas, utilizadas na edição do modelo e na implementação de uma aplicação de teste, foram o editor de ontologias Protégé e a API do arcabouço Jena ${ }^{9}$. Foram adotadas como referência para representação semântica as ontologias $O W L$-Time e WGS84, apresentadas na Seção 2.2. Dessa forma, o modelo ontológico utilizado consiste na combinação de semântica espacial e temporal para a representação semântica de informações espaço-temporais.

Nesse contexto, a trajetória de uma linha de ônibus é constituída pela ligação entre uma sequência de pontos espaciais, que possuem latitude e longitude. Cada linha possui diversos pontos no decorrer da sua extensão, para embarque e desembarque de passageiros, onde cada ponto tem sua localização espacial conhecida. Uma linha também conta com um determinado número de ônibus, que varia de acordo com a demanda e o tempo de espera da linha. Cada ônibus possui o seu identificador e tem sua localização monitorada constantemente, via GPS, dentro do seu trajeto na linha.

O Exemplo 1, a seguir, apresenta a definição da classe Point, que é subclasse de SpatialThing. Esta classe possui as propriedades de localização latitude (lat) e longitude (long), entre outras, importadas pelo modelo OntoBus.

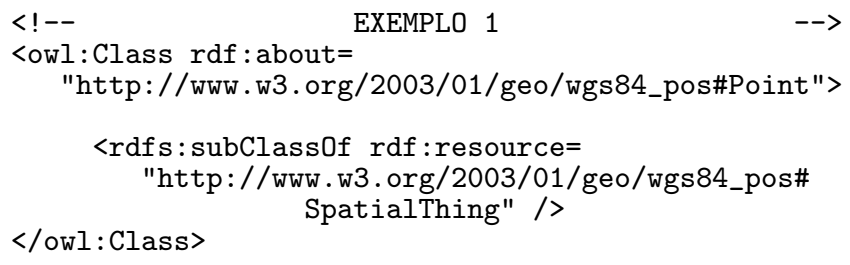

A frota de uma linha deve circular respeitando os horários e o tempo de espera definidos pelo órgão gerenciador. Os horários de circulação e a frequência de ônibus, mesmo dentro de uma linha específica, variam, tendo como critérios: i) dia útil, ii) sábado, e iii) domingo e feriado. As informações temporais mais relevantes, neste domínio, são o horário em que um ônibus chega a um ponto e, quando este se encontra em movimento pelo trajeto, qual o tempo gasto

\footnotetext{
${ }^{9}$ Apache Jena: https://jena. apache.org/
}

de sua localização atual até um ponto em específico. Outras informações temporais são o tempo gasto no trajeto total de uma linha, ou entre dois pontos específicos, entre outras.

As principais instâncias de semântica temporal utilizadas no modelo OntoBus, são realizadas a partir das classes Instant e Interval, cuja definição ontológica é apresentada no Exemplo 2.

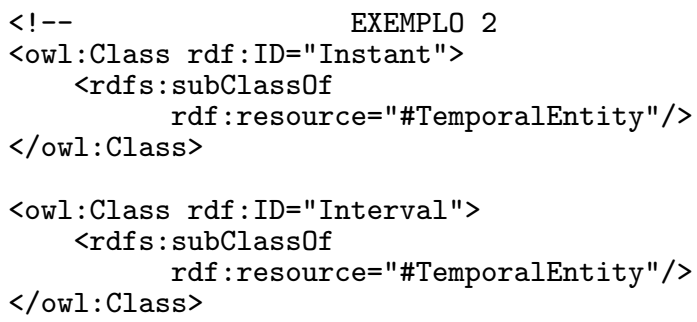

Para modelar os dados em questão, foram utilizadas as classes e propriedades da ontologia OWL-Time, declarada no modelo pelo prefixo time: (linha 1 - Exemplo 3) e do vocabulário $W G S 84$, por geo: (linha 2). Estas classes e propriedades permitem modelar a evolução de uma entidade ao longo do tempo e no espaço (através de coordenadas geográficas). Neste caso, o horário que o ônibus passou por cada um dos pontos é registrado na forma de uma tripla no modelo (linhas 17 a 21).

$\#$
1
2
3
4
5
6
7
8
9
10
11
12
13
1
15
16
17
18
19
20
21

EXEMPLO 3 Oprefix time: <http://www.w3.org/2006/time\#> .
Oprefix geo:

<http://www.w3.org/2003/01/geo/wgs84_pos\#> . Oprefix owl: <http://www.w3.org/2002/07/owl\#>

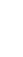
].

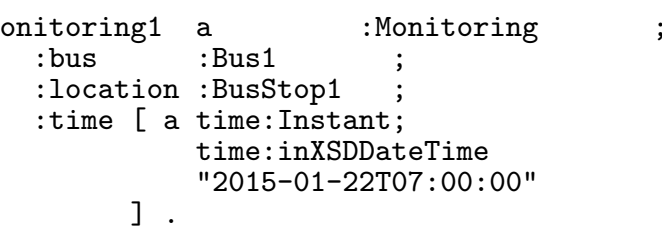

O modelo OntoBus, além da modelagem espaço-temporal advinda das ontologias apresentadas, fornece a modelagem relacionada ao gerenciamento das informações de transporte coletivo. As principais classes neste domínio são:

- Bus: esta classe descreve um ônibus, tendo como propriedades a linha cumprida por este e o seu identificador; a localização espacial de um ônibus também pode ser especificada, se necessário;

- BusStop: descreve um ponto (parada) de ônibus, tendo como principais propriedades a sua localização (latitude e longitude) e o identificador, ou nome do ponto; 

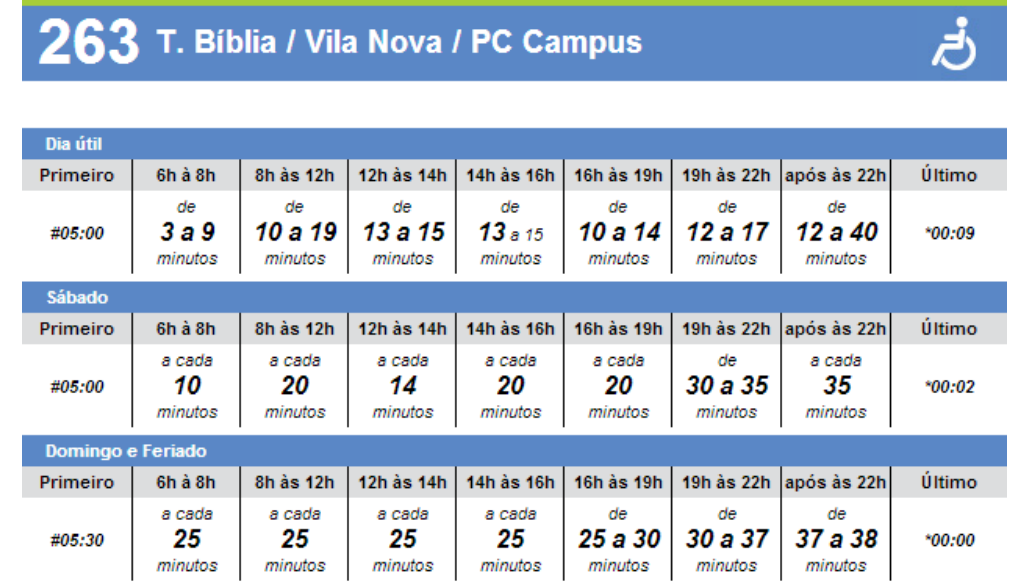

(a) Informação temporal.

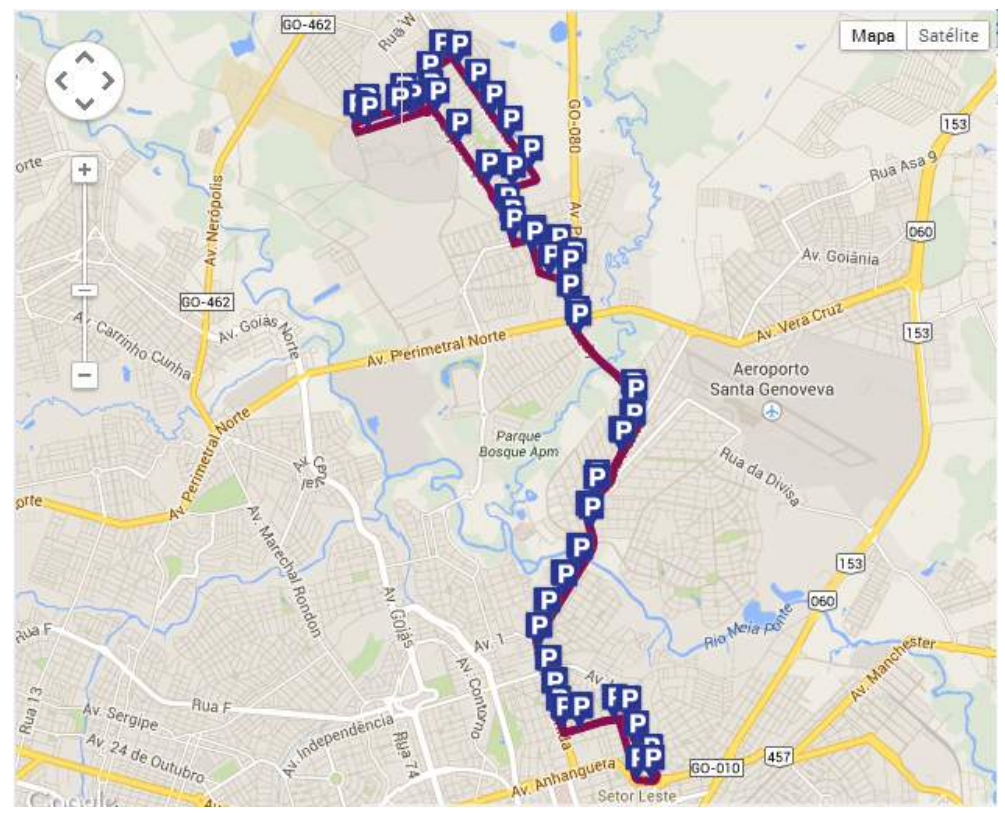

(b) Informação espacial.

Figura 4: Tabela de horários e mapa com os pontos de parada da linha 263.

- Monitoring: classe que relaciona um Bus a um BusStop, registrando o horário que um ônibus passou por um determinado ponto, ou também os horários previstos.

No Exemplo 3 são apresentados trechos de instâncias que utilizam o modelo OntoBus, no caso, a evolução da entidade Bus1 da classe Bus (linha 5) em relação às entidades da classe BusStop (linha 6). Este trecho também apresenta a declaração das entidades utilizadas e a associação das informações temporais (instante em que o ônibus estava em um ponto) e espaciais (latitude e longitude de um determinado ponto de ônibus) à entidade Bus1, que representa um ônibus da linha 263.

Nas linhas 5 a 7 são declaradas as classes Bus, BusStop e Monitoring, de acordo com a linguagem OWL. Em seguida, na linha 8 , é criado um indivíduo Bus1 do tipo Bus, que possui o id 263_id, como um determinado ônibus para este exemplo. Também é criado um indivíduo BusStop1, do tipo
BusStop, na linha 10, cuja propriedade nome possui o valor 31502 , que é o identificador do ponto de ônibus em questão. O indivíduo BusStop1 possui ainda sua localização, através das propriedades latitude e longitude, da classe Point, importada pelo OntoBus do vocabulário WGS84.

Os nomes de pontos de ônibus citados neste e nos demais exemplos apresentados são os identificadores reais da linha 263. Todos os pontos de ônibus foram devidamente representadas utilizando o modelo OntoBus. Um padrão foi adotado para o identificador do ônibus (nomeDaLinha_idOnibus), sendo 263_id adotado na maioria dos exemplos, uma vez que diferentes ônibus participam da frota desta linha, e podem ser também remanejados de/para outras.

Em seguida, a partir da linha 16 do Exemplo 3, é realizada a associação da instância Bus1 ao ponto BusStop1, onde é também registrado o momento exato em que o ônibus esteve no ponto, através da classe Instant, importada da ontologia 
OWL-Time. A mesma modelagem é feita para os demais pontos de ônibus (BusStop2, BusStop3...) e também para os demais ônibus que percorrem a linha (Bus2, Bus3...).

Este trecho mostra a representação de um determinado ônibus (263_idBus), que passa pelo ponto (31502) em um momento específico (dia 22/01/2015, às 7 horas da manhã). Como a linha 263 possui 64 pontos de parada, exemplificamos somente este trecho, que representa o primeiro ponto de parada da linha, localizada em um dos maiores terminais da cidade. As consultas, que serão apresentadas posteriormente, foram realizadas sobre a representação completa da linha 263 e, portanto, trazem os resultados para todas os pontos de parada da linha.

\subsection{Validação da Abordagem}

Finalizada a etapa de representação, é iniciada a etapa de armazenamento dos modelos em repositórios específicos para triplas. Com o modelo ontológico e suas instâncias, sejam em memória ou armazenadas no repositório, diversos tipos de consultas puderam ser realizadas. A seguir, um exemplo de consulta que retorna o horário que os ônibus da linha 263 estiveram em cada ponto de parada da linha, bem como a sua localização espacial.

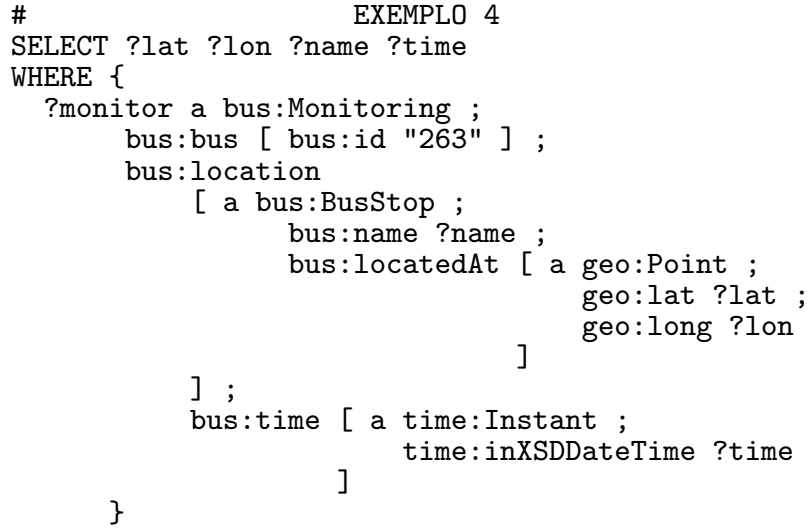

Esta consulta utiliza todos os elementos armazenados e busca todas as triplas que representam a evolução da entidade ônibus, representada pela variável ?monitor, onde: 1) o id do ônibus é $263 ; 2$ ) a entidade ônibus possui localização, dada pelo nome do ponto de ônibus (?name), sua latitude (?lat) e longitude (?lon); e o instante em que entidade ônibus esteve no ponto especificado (?time). O resultado retornado pela consulta é mostrado a seguir.

\begin{tabular}{|c|c|c|c|}
\hline lat & on & I name & I time \\
\hline & & $:=====$ & $=======$ \\
\hline "-16.671581" & | "-49.235329" & | "31502" & | "2014-05-22T07:00:00" \\
\hline$"-16$ & | $"-49.2$ & | "315 & | "2014-05-22 \\
\hline$"-16.665117 "$ & | " -49.239 & | "4650" & | "2014-05-22 \\
\hline$\cdots$ & $\cdots$ & . & $\cdots$ \\
\hline .. & .. & $\ldots$ & . \\
\hline$"-16.665421 "$ & | "-49.237709" & | "2917" & | "2014-05-22T08:32:00" \\
\hline | "-16.667955" & | "-49.236900" & | "2915" & | "2014-05-22T08:33:30" \\
\hline$"-16.670416 "$ & $"-49.235402 "$ & | "2913" & I "2014-05-22T08:35:00" \\
\hline
\end{tabular}

Outras consultas também foram implementadas para validação do modelo OntoBus e da representação da linha 263 , como o tempo gasto no percurso completo e entre dois pontos distintos da linha, entre outras.

\subsection{Análise dos Resultados}

A partir da abordagem realizada, além da representação ontológica das informações de uma rede de trasporte coletivo, com ênfase para as informações espaço-temporais, foi possível criar um repositório de dados que fornece o histórico da linha de ônibus que foi objeto de estudo. Este repositório é alimentado pelas informações de cada linha e de seus componentes (ônibus e paradas de ônibus), além das informações de contexto, como horários e localização.

O histórico dos dados armazenados permite ainda realizar o acompanhamento da evolução espacial e temporal das entidades ônibus e determinar quais ônibus estiveram em cada parada em determinado momento ou intervalo do tempo. Dessa forma, consultas podem ser realizadas para determinar quais ônibus atrasaram (ou adiantaram) em suas chegadas aos pontos de parada e terminais, de acordo com seus horários previstos de chegada.

Desses dados ainda podem ser extraídos padrões de comportamento por meio de técnicas de mineração de dados. Esse tipo de informação é de grande relevância para órgãos que gerenciam o sistema de transporte como um diagnóstico do serviço oferecido para que possíveis falhas sejam detectadas e corrigidas.

A representação semântica permite ainda, como uma de suas principais vantagens, o raciocínio sobre os modelos e as relações entre os dados. A partir disso, podem ser realizadas inferências como: a saída de um ônibus de sua trajetória; a parada em um ponto não determinado na rota; a não alimentação (ou anormalidades) em uma linha, conforme os horários pré-estabelecidos, dentre outras inferências.

\section{TRABALHOS RELACIONADOS}

Segundo Harbelot [5] é necessário considerar três componentes para a representação de uma entidade no projeto de um sistema de conhecimento espaço-temporal: 1) componente espacial, que consiste na localização e/ou geometria de uma entidade; 2) temporal, que define o intervalo da existência de uma entidade; e a 3) semântica, que define um significado para cada entidade, além do significado puramente espacial e temporal. Estes componentes são utilizados na construção do modelo proposto neste trabalho.

Um dos principais desafios para o desenvolvimento de cidades inteligentes é o provimento eficiente de transporte público. Neste sentido, os Serviços de Transporte Inteligente são a principal tecnologia de informação e comunicação utilizada como solução computacional neste domínio. Segundo [12], diversos aspectos têm sido abordados para esta finalidade, tais como: ( $i$ ) simuladores de tráfego/trânsito, (ii) ferramentas que oferecem informação online sobre tráfego e trânsito em geral, e (iii) ferramentas e sistemas para gerenciamento de transporte público especialmente voltadas para empresas. Utilizando os conceitos de espaço e de tempo, sejam direta ou indiretamente, diversos sistemas têm sido propostos para as finalidades apresentadas.

O OneBusAway [3] é um serviço desenvolvido pela Universidade de Washington, que consiste em um conjunto de ferramentas para fornecer dados sobre as chegada e partidas de ônibus na cidade de Seattle. O objetivo deste sistema é ajudar passageiros que utilizam os ônibus da cidade como meio de transporte, mostrando a partir do momento da consulta as informações solicitadas com os horários atualizados. Estas informações são fornecidas diretamente pela agência 
oficial de transportes de Seattle, que oferece os dados extraídos em tempo real dos ônibus da cidade, que são equipados com GPS.

Neste mesmo intuito, no Brasil, diversos sistemas móveis são oferecidos para usuários do transporte coletivo urbano. Em São Paulo, o Olho Vivo ${ }^{10}$ é um sistema que permite a visualização em tempo real a localização dos ônibus na cidade e obter a informação do tempo gasto para chegar em determinado ponto de parada. Em Goiânia, a Rede Metropolitana de Transporte Coletivo (RMTC) disponibiliza um serviço parecido, chamado Olho no Ônibus ${ }^{11}$, serviço que também é oferecido por aplicativos como o GynBus ${ }^{12}$, que permite a consulta de horários por linhas e pontos de ônibus. Outras capitais brasileiras, como Porto Alegre, Florianópolis e Rio de Janeiro também oferecem serviços deste tipo.

Existem ainda ferramentas para monitoramento de veículos em geral, como o Google Traffic, que é um serviço gratuito que pode ser utilizado para acompanhar o comportamento do tráfego urbano. As informações oferecidas por este serviço são oferecidas implicitamente por usuários, que enviam sua posição atual e velocidade para a Google quando utilizam seus serviços em dispositivos móveis. Outros tipos de ferramentas a serem destacadas são as voltadas para o entendimento e gerenciamento do transporte público, cuja operação e manutenção ainda é inviável para muitas cidades brasileiras [12].

A proposta apresentada neste trabalho difere das demais por explorar as características de um modelo ontológico, que carrega consigo maior expressividade, interoperabilidade e poder de inferência. A utilização de um modelo semântico para o gerenciamento de informações espaço-temporais do trânsito e do transporte coletivo urbano permite explorar informações implícitas através das relações entre as informações coletadas. Tais informações são inferidas por software, e a complexidade das relações entre estas informações está totalmente representada no modelo ontológico, minimizando a complexidade no desenvolvimento de aplicações.

\section{CONCLUSÕES}

Apresentamos neste trabalho uma abordagem ontológica para a representação de informações relacionadas ao gerenciamento de redes de transporte coletivo, bem como o armazenamento e a consulta destas informações utilizando tecnologias da Web Semântica. Como principais contribuições destacamos a utilização do histórico de dados semânticos e a realização de inferências para o levantamento e extração de informações relevantes neste contexto.

O modelo proposto pode ser utilizado por diferentes aplicações, uma vez que a padronização da representação semântica garante a interoperabilidade de dados e a validação dos mesmos quanto ao domínio. O modelo OntoBus ainda não é considerado totalmente completo, mas pode ser importado e evoluído nas próprias aplicações ou em outros modelos.

Destacamos como trabalho futuro a representação de outras linhas de ônibus, no intuito de criar um repositório semântico da rede metropolitana de transporte coletivo, que pode ser utilizado por diferentes sistemas de transporte inteligente. Também se faz necessária a implementação de uma

\footnotetext{
${ }^{10}$ Olho Vivo: http://olhovivo.sptrans.com.br/

${ }^{11}$ Olho no Ônibus: http://www.rmtcgoiania.com.br/ olho-no-onibus

${ }^{12}$ GynBus: http: //www.gynbus.com.br/
}

interface gráfica de usuário e/ou de uma aplicação móvel para acesso às informações modeladas, melhorando a aplicação apresentada como prova de conceito.

Além disso, é válida a possibilidade de integração com o sistema de disponibilização de informações de localização de ônibus em tempo real, da rede municipal de transporte coletivo. Esta integração, aliada ao repositório semântico, permitiria a exploração de informações que permitem fornecer informações a aplicações de usuários, e explorar ainda melhor o poder de expressividade e inferência do modelo ontológico apresentado neste trabalho.

\section{REFERÊNCIAS}

[1] S. Batsakis and E. G. Petrakis. Sowl: spatio-temporal representation, reasoning and querying over the semantic web. In Proceedings of the 6th International Conference on Semantic Systems, page 15. ACM, 2010.

[2] B. DuCharme. Learning Sparql. "O'Reilly Media, Inc.", 2013.

[3] B. Ferris, K. Watkins, and A. Borning. Location-aware tools for improving public transit usability. Pervasive Computing, IEEE, 9(1):13-19, Jan 2010.

[4] B. Harbelot, H. Arenas, C. Cruz, et al. Continuum: A spatiotemporal data model to represent and qualify filiation relationships. In 4 th ACM SIGSPATIAL International Workshop on GeoStreaming (IWGS) 2013, 2013.

[5] B. Harbelot, H. Arenas, C. Cruz, et al. The spatio-temporal semantics from a perdurantism perspective. In In Proceedings of the Fifth International Conference on Advanced Geographic Information Systems, Applications, and Services GEOProcessing, 2013.

[6] J. Hebeler, M. Fisher, R. Blace, and A. Perez-Lopez. Semantic web programming. John Wiley \& Sons, 2011.

[7] J. R. Hobbs and F. Pan. An Ontology of Time for the Semantic Web. ACM Transactions on Asian Language Information Processing, 3:66-85, 2004.

[8] J. R. Hobbs and F. Pan. Time Ontology in OWL. http://www.w3.org/TR/owl-time/, último acesso em Maio de 2014, 2013.

[9] D. F. B. Leite, J. H. Rocha, and C. S. Baptista. Busão: um Sistema de Informações Móvel para Auxílio à Mobilidade Urbana Através do Uso de Transporte Coletivo. In IX Simpósio Brasileiro de Sistemas de Informação, pages 170-181. 2013.

[10] N. Noy and D. L. McGuinness. Ontology development 101. KS Laboratory, Stanford University, 2001.

[11] L. E. Santos and R. Girardi. Uma técnica orientada por objetivos para a construção de ontologias de aplicação. In VIII Simpósio Brasileiro de Sistemas de Informação, pages 13-24, 2012.

[12] T. L. T. Silveira and M. Pasin. Provimento de informações sobre transporte público urbano para empresas concessionárias: Simulação e avaliação apoiada por um sistema multi-agentes. In X Simpósio Brasileiro de Sistemas de Informação, pages 565-576, 2014.

[13] W3C. Basic Geo (WGS84 lat/long) Vocabulary. http://www.w3.org/2003/01/geo/, último acesso em Maio de 2014, 2013. 